University of Nebraska - Lincoln

DigitalCommons@University of Nebraska - Lincoln

Faculty Publications from the Department of Electrical \& Computer Engineering, Department Electrical and Computer Engineering

10-1997

\title{
Frequency-Mixing Detection of Polarization-Modulated Light Using Different Periodic Analyzers
}

R.M.A. Azzam

Follow this and additional works at: https://digitalcommons.unl.edu/electricalengineeringfacpub

Part of the Computer Engineering Commons, and the Electrical and Computer Engineering Commons

This Article is brought to you for free and open access by the Electrical \& Computer Engineering, Department of at DigitalCommons@University of Nebraska - Lincoln. It has been accepted for inclusion in Faculty Publications from the Department of Electrical and Computer Engineering by an authorized administrator of DigitalCommons@University of Nebraska - Lincoln. 


\title{
Frequency-Mixing Detection of Polarization-Modulated Light Using Different Periodic Analyzers
}

\author{
R. M. A. Azzam \\ Hematology Division and Department of Internal Medicine, \\ University of Nebraska Medical Center, Omaha, Nebraska 68105 \\ and Electrical Materials Laboratory, College of Engineering, \\ University of Nebraska, Lincoln, Nebraska 68588
}

\begin{abstract}
We derive the equations that permit the use of six different periodic analyzers for the frequency-mixing detection (FMD) of polarization-modulated light. The six periodic analyzers are (1) the rotating linear analyzer, (2) rotating half-wave plate and fixed linear analyzer, (3) rotating quarter-wave plate and fixed linear analyzer, (4) rotating linear analyzer and fixed linear analyzer, (5) oscillating-phase retarder and fixed linear analyzer, and (6) oscillating optical rotator and fixed linear analyzer. In all cases, the average polarization and the polarization and intensity modulation parameters of the light beam can be determined easily and explicitly from limited Fourier analysis of the detected signal.
\end{abstract}

\section{Introduction}

In lightwave communication systems information may be imparted to the light beam in the form of polarization modulation. ${ }^{1}$ Polarization modulation is also encountered in "modulation spectroscopy" where a light beam interacts with a material sample (e.g., a crystal) subjected to an alternating perturbation such as an electric, magnetic or stress field. ${ }^{2}$ In these and other related applications it is important to detect a modulated state of polarization.

We have recently suggested that the parameters that characterize the polarization- and intensity-modulated light be measured by what we call frequency-mixing detection (or FMD). ${ }^{3}$ In FMD, a light beam modulated at a frequency $\omega_{\mathrm{m}}$ passes through a periodic optical analyzer that oscillates at a different frequency $\omega_{\mathrm{a}}\left(\omega_{\mathrm{a}}<<\omega_{\mathrm{m}}\right)$. The resulting interaction causes frequency mixing which is borne in variations of the intensity of the light leaving the analyzer. Fourier analysis of the signal received by a photodetector placed after the analyzer allows the determination of both the average polarization and the polarization and intensity modulation of the beam.

Let the light beam be described by its total intensity I and its ellipse of polarization specified by the complex number ${ }^{4}$

$$
\begin{aligned}
\chi & =\tan \psi e^{j \Delta} \\
& =E_{y} / E_{X},
\end{aligned}
$$

where $E_{X}$ and $E_{y}$ are the components of the light electric vector along two mutually orthogonal transverse axes $x$ and $y$. Beam modulation is assumed to be small and sinusoidal of frequency $\omega_{\mathrm{m}}$ :

$$
\mathrm{x}=\overline{\mathrm{x}}+\hat{\delta} \hat{\mathrm{x}} \cos \omega_{\mathrm{m}} \mathrm{t}, \mathrm{x}=\mathrm{I}, \psi, \Delta .
$$

\footnotetext{
*This paper is a revision of one presented at the SPIE seminar on Polarized Light, Aug. 24, 25, 1976, San Diego, California, and published in the Proceedings of that meeting, SPIE Vol. 88, 1976.
}

1384 received August 5, 1976.
The analyzer is characterized by a set of parameters $\alpha_{j}(j=1$, $2, .$.$) one or more of which are modulated (mechanically, electo-$ optically, or otherwise) at a frequency $\omega_{\mathrm{a}}$. The intensity of the light transmitted by the analyzer can be expressed as

$$
\mathrm{I}_{\mathrm{t}}=\text { const } \operatorname{If}\left(\psi, \Delta, \alpha_{\mathrm{j}}\right) \text {, }
$$

where $\mathrm{f}$ is a function whose form depends on the analyzer. When $I_{t}$ is linearly detected by a polarization-independent photodetector, the total signal consists of two parts

$$
\mathrm{S}_{\mathrm{t}}=\mathrm{S}_{\mathrm{bb}}+\delta \mathrm{S}_{\mathrm{mc}} \text {. }
$$

In Eq. (5) $\mathrm{S}_{\mathrm{bb}}$ is a periodic baseband signal of fundamental frequency $\omega_{\mathrm{a}}$ whose waveform is that of $\mathrm{f}$ :

$$
\mathrm{S}_{\mathrm{bb}}=\text { const } \overline{\mathrm{I}} \sum_{\mathrm{m}=0}^{\infty} \mathrm{f}_{\mathrm{m}} \sin \left(\mathrm{m} \omega_{\mathrm{a}} \mathrm{t}+\theta_{\mathrm{m}}\right) \text {. }
$$

The Fourier amplitude $\left(\mathrm{f}_{\mathrm{m}}\right)$ and phase $\left(\theta_{\mathrm{m}}\right)$ coefficients in Eq. (6) are functions of the average polarization parameters $\bar{\psi}, \triangle$ and the time-independent parts of the analyzer parameters $\alpha_{j}$. $\delta \mathrm{S}_{\mathrm{mc}}$ is an amplitude-modulated-carrier small signal

$$
\delta \mathrm{S}_{\mathrm{mc}}=\delta \mathrm{S}_{\mathrm{e}} \cos \omega_{\mathrm{m}} \mathrm{t}
$$

of center (carrier) frequency $\omega_{\mathrm{m}}$ and a periodic envelope $\delta \mathrm{S}_{\mathrm{e}}$ of fundamental frequency $\omega_{\mathrm{a}}$ :

$$
\begin{aligned}
& \delta \mathrm{S}_{\mathrm{e}}=\operatorname{const} \overline{\mathrm{I}}\left[\left(\hat{\delta \mathrm{I} / \overline{\mathrm{I}})} \sum_{\mathrm{m}=0}^{\infty} \mathrm{f}_{\mathrm{m}} \sin \left(\mathrm{m} \omega_{\mathrm{a}} \mathrm{t}+\theta_{\mathrm{m}}\right)+\right.\right. \\
& \hat{\delta \psi} \sum_{\mathrm{m}=0}^{\infty} \mathrm{f}_{\psi \mathrm{m}} \sin \left(\mathrm{m} \omega_{\mathrm{a}} \mathrm{t}+\theta_{\psi \mathrm{m}}\right)+ \\
& \left.\hat{\delta \Delta} \sum_{\mathrm{m}=0}^{\infty} \mathrm{f}_{\Delta \mathrm{m}} \sin \left(\mathrm{m} \omega_{\mathrm{a}} \mathrm{t}+\theta_{\Delta \mathrm{m}}\right)\right] .
\end{aligned}
$$

The infinite-Fourier-series coefficients of $\hat{\delta} \psi$ and $\hat{\delta} \Delta$ in the righthand side of Eq. (8) represent the periodic partial derivatives of the analyzer function $\mathrm{f}$ with respect to $\psi$ and $\Delta$ respectively;

$$
\mathrm{f}_{\psi}=\partial \mathrm{f} / \partial \psi, \mathrm{f}_{\triangle}=\partial \mathrm{f} / \partial \Delta \text {. }
$$

The average polarization $\bar{\psi}, \bar{\Delta}$ can be determined by measuring the normalized amplitudes of the $\operatorname{cosp} \omega_{\mathrm{a}} \mathrm{t}$ and $\operatorname{sinp} \omega_{\mathrm{a}} \mathrm{t}$ components of the same $\mathrm{p}$ harmonic of the baseband signal $\mathrm{S}_{\mathrm{bb}}$ and using the following equations

$$
\eta_{\mathrm{p} \omega \mathrm{a}}^{\mathrm{c}}=\mathrm{f}_{\mathrm{p}} \sin \theta \mathrm{p} / \mathrm{f}_{\mathrm{o}}, \eta_{\mathrm{p} \omega \mathrm{a}}^{\mathrm{s}}=\mathrm{f}_{\mathrm{p}} \cos \theta_{\mathrm{p}} / \mathrm{f}_{\mathrm{o}} .
$$

Alternatively, $\bar{\psi}, \bar{\Delta}$ can be determined from the normalized amplitudes $\left(\eta_{\mathrm{p} \omega \mathrm{a}}, \eta_{\mathrm{q} \omega \mathrm{a}}\right)$ of two different frequency components $\mathrm{p}$ and $\mathrm{q}$ of $\mathrm{S}_{\mathrm{bb}}$ using 


$$
\eta_{\mathrm{p} \omega \mathrm{a}}=\mathrm{f}_{\mathrm{p}} / \mathrm{f}_{\mathrm{o}}, \eta_{\mathrm{q} \omega \mathrm{a}}=\mathrm{f}_{\mathrm{q}} / \mathrm{f}_{\mathrm{o}}
$$

The modulation parameters $\hat{\delta \mathrm{I}} / \overline{\mathrm{I}}, \hat{\delta} \psi$ and $\hat{\delta \Delta}$ are determined by measuring the normalized dc component $\left(\eta^{\mathrm{e}}{ }_{\mathrm{o}}\right)$ and the normalized amplitudes

$$
\left(\eta_{\mathrm{p} \omega \mathrm{a}}^{\mathrm{ec}}, \eta_{\mathrm{p} \omega \mathrm{a}}^{\mathrm{es}}\right)
$$

of the $\operatorname{cosp} \omega_{\mathrm{a}} \mathrm{t}$ and $\operatorname{sinp} \omega_{\mathrm{a}} \mathrm{t}$ components of the same $\mathrm{p}$ harmonic of the envelope signal $\delta \mathrm{S}_{\mathrm{e}}$ and using the following three linear algebraic equations

$$
\begin{aligned}
& \eta_{\mathrm{o}}^{\mathrm{e}}=(\hat{\delta \mathrm{I}} / \overline{\mathrm{I}})+\left(\mathrm{f}_{\psi \mathrm{o}} / \mathrm{f}_{\mathrm{o}}\right) \hat{\psi} \psi+\left(\mathrm{f}_{\Delta \mathrm{o}} / \mathrm{f}_{\mathrm{o}}\right) \hat{\delta \Delta}, \\
& \eta_{\mathrm{p} \omega \mathrm{a}}^{\mathrm{ec}}=\left(\mathrm{f}_{\mathrm{p}} \sin \theta_{\mathrm{p}} / \mathrm{f}_{\mathrm{o}}\right)(\hat{\delta \mathrm{I} / \overline{\mathrm{I}})})+\left(\mathrm{f}_{\psi \mathrm{p}} \sin \theta_{\psi \mathrm{p}} / \mathrm{f}_{\mathrm{o}}\right) \hat{\delta} \psi+ \\
& \left(\mathrm{f}_{\Delta \mathrm{p}} \sin \theta \Delta \mathrm{p} / \mathrm{f}_{\mathrm{o}}\right) \hat{\Delta} \text {, } \\
& \eta_{\mathrm{p} \omega \mathrm{a}}^{\mathrm{es}}=\left(\mathrm{f}_{\mathrm{p}} \cos \theta_{\mathrm{p}} / \mathrm{f}_{\mathrm{o}}\right)(\hat{\delta \mathrm{I} / \overline{\mathrm{I}}})+\left(\mathrm{f}_{\psi \mathrm{p}} \cos \theta_{\psi \mathrm{p}} / \mathrm{f}_{\mathrm{o}}\right) \hat{\delta} \psi+ \\
& \left(\mathrm{f}_{\Delta \mathrm{p}} \cos \theta_{\Delta \mathrm{p}} / \mathrm{f}_{\mathrm{o}}\right) \hat{\delta} \Delta \text {. }
\end{aligned}
$$

Alternatively, the same modulation parameters may be determined from the normalized amplitudes of the carrier $\left(\omega_{m}\right)$ and two nonzero sidebands $\left(\omega_{\mathrm{m}} \pm \mathrm{p} \omega_{\mathrm{a}}, \omega_{\mathrm{m}} \pm \mathrm{q} \omega_{\mathrm{a}}\right)$ of $\delta \mathrm{S}_{\mathrm{mc}}$ provided that the harmonics of $f, f_{\psi}$ and $f_{\triangle}$ of frequency $p \omega_{a}$ or $q \omega_{a}$ are either in phase or out of phase among themselves. The equations that apply in this case are

$$
\begin{aligned}
& \eta_{\omega_{\mathrm{m}}}=\left(\hat{\delta \mathrm{I} / \overline{\mathrm{I}})}+\left(\mathrm{f}_{\psi_{\mathrm{o}}} / \mathrm{f}_{\mathrm{o}}\right) \hat{\delta} \dot{\psi}+\left(\mathrm{f}_{\Delta \mathrm{o}} / \mathrm{f}_{\mathrm{o}}\right) \hat{\delta} \Delta,\right. \\
& 2 \eta_{\omega_{\mathrm{m}}} \pm \mathrm{p} \omega_{\mathrm{a}}=\left(\mathrm{f}_{\mathrm{p}} / \mathrm{f}_{\mathrm{o}}\right)\left(\hat{\delta \mathrm{I} / \overline{\mathrm{I}})}+\left(\mathrm{f}_{\psi \mathrm{p}} / \mathrm{f}_{\mathrm{o}}\right) \hat{\delta} \dot{\psi}+\left(\mathrm{f}_{\Delta \mathrm{p}} / \mathrm{f}_{\mathrm{o}}\right) \hat{\delta \Delta},\right. \\
& 2 \eta \omega_{\mathrm{m}} \pm \mathrm{q} \omega_{\mathrm{a}}=\left(\mathrm{f}_{\mathrm{q}} / \mathrm{f}_{\mathrm{o}}\right)\left(\hat{\delta \mathrm{I} / \overline{\mathrm{I}})}+\left(\mathrm{f}_{\psi \mathrm{q}} / \mathrm{f}_{\mathrm{o}}\right) \hat{\delta} \psi+\left(\mathrm{f}_{\Delta \mathrm{q}} / \mathrm{f}_{\mathrm{o}}\right) \hat{\delta \Delta}\right. \text {. }
\end{aligned}
$$

In Eqs. (10) and (11) all of the amplitudes $\eta$ 's are normalized with respect to the dc component of the baseband signal $\mathrm{S}_{\mathrm{bb}}$, which is also equal to the dc component of the total signal $S_{t}$.

Now that we have briefly reviewed the fundamental equations of FMD, we examine six special cases of six different periodic analyzers. The six periodic analyzers are (1) the rotating linear analyzer, ${ }^{5-8}(2)$ rotating half-wave plate and fixed linear analyzer, ${ }^{9}(3)$ rotating quarter-wave plate and fixed linear analyzer, ${ }^{10}(4)$ rotating linear analyzer and fixed linear analyzer, ${ }^{11}$ (5) oscillating-phase retarder and fixed linear analyzer, ${ }^{12,13}$ and (6) oscillating optical rotator and fixed linear analyzer. ${ }^{14}$ All of these periodic analyzers have been previously used for the measurement of unmodulated polarization states only. The results in this special case constitute a by-product of the present paper, assuming totally polarized light.

\section{The Two-Element Periodic Analyzer}

Figure 1 shows a two-element periodic analyzer consisting of a

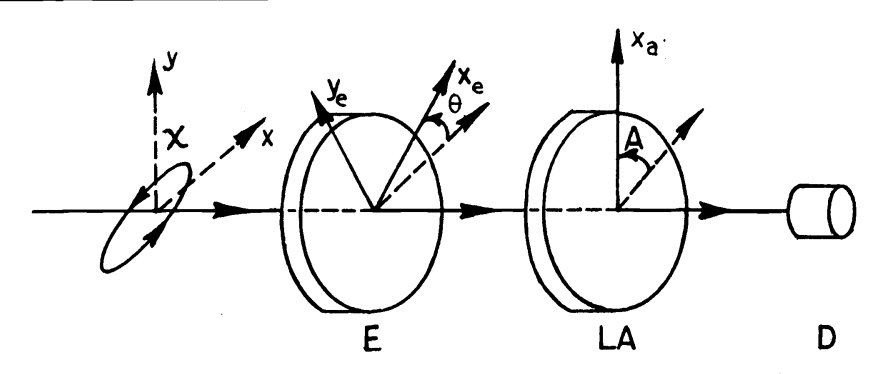

Figure 1. The two-element periodic analyzer. linear analyzer LA preceded by an optical element $\mathrm{E}$ characterized by two principal axes $x_{e}$ and $y_{e}$ and the ratio $\tau e^{-j \delta}$ of the complex amplitude transmittance along the $\mathrm{y}_{\mathrm{e}}$ axis to that along the $x_{e}$ axis. The orientation of LA around the beam is specified by the azimuth angle $A$ of the transmission axis $x_{a}$, while the orientation of $E$ is specified by the azimuth angle $\theta$ of the fast axis $x_{e}$. Both $A$ and $\theta$ are measured from the $\mathrm{x}$ axis of a transverse reference coordinate system $x y$.

When the light beam, described by its total intensity I and its ellipse of polarization represented by the complex number $\chi$ [Eq. (1)], passes through the two-element analyzer, the transmitted intensity can be put in the form of Eq. (4), where*

$$
\begin{aligned}
& \mathrm{f}=1+\mu_{1} \cos 2 \psi \cos 2 \theta+\mu_{1} \sin 2 \psi \cos \Delta \sin 2 \theta+ \\
& \mu_{2} \sin 2 \psi \cos \Delta \cos 2 \theta \cos \delta-\mu_{2} \cos 2 \psi \sin 2 \theta \cos \delta+ \\
& \mu_{2} \sin 2 \psi \sin \Delta \sin \delta,
\end{aligned}
$$

and

$$
\begin{aligned}
& \mu_{1}=\left[1-\tau^{2} \tan ^{2}(\mathrm{~A}-\theta)\right] /\left[1+\tau^{2} \tan ^{2}(\mathrm{~A}-\theta)\right], \\
& \mu_{2}=2 \tau \tan (\mathrm{A}-\theta) /\left[1+\tau^{2} \tan ^{2}(\mathrm{~A}-\theta)\right] .
\end{aligned}
$$

We restrict our interest to the case when $\tau=1$ (i.e., the element $E$ exhibits pure retardation only, but no differential absorption or dichroism). With $\tau=1, \mu_{1}$ and $\mu_{2}$ of Eqs. (13a) simplify to

$$
\mu_{1}=\cos (2 \mathrm{~A}-2 \theta), \mu_{2}=\sin (2 \mathrm{~A}-2 \theta) \text {, }
$$

and Eq. (12) now reads

$$
\begin{aligned}
\mathrm{f}=1 & +\cos 2 \psi[\cos (2 \mathrm{~A}-2 \theta) \cos 2 \theta-\sin (2 \mathrm{~A}-2 \theta) \sin 2 \theta \cos \delta] \\
& +\sin 2 \psi \cos \triangle[\sin (2 \mathrm{~A}-2 \theta) \cos 2 \theta \cos \delta+\cos (2 \mathrm{~A}-2 \theta) \sin 2 \theta] \\
& +\sin 2 \psi \sin \triangle \sin (2 \mathrm{~A}-2 \theta) \sin \delta .
\end{aligned}
$$

The use of several periodic analyzers for the frequency-mixing detection of polarization-modulated light will be discussed in the following sections based on Eq. (14) and the review of Section I.

\section{Rotating Linear Analyzer (RLA)}

If we set $\delta=\theta=0$ in Eq. (14), we obtain

$$
f=1+\cos 2 \psi \cos 2 A+\sin 2 \psi \cos \Delta \sin 2 A,
$$

which represents the case when the element $E$ is absent and only the linear analyzer LA is present. The function $f$ of Eq. (15) differs from the corresponding function given by Eq. (23) of Ref. 3 by the multiplicative factor $\cos ^{2} \psi$ which is missing in the latter equation. We redo the derivations in this case based on the correct function $f$ of Eq. (15). If we set $A=1 / 2 \omega_{\mathrm{a}} t$ in Eq. (15), and take the first partial derivatives of the result with respect to $\psi$ and $\Delta$, we obtain

$$
\begin{aligned}
& \mathrm{f}=1+\cos 2 \psi \cos \omega_{\mathrm{a}} \mathrm{t}+\sin 2 \psi \cos \Delta \sin \omega_{\mathrm{a}} \mathrm{t}, \\
& \mathrm{f}_{\psi}=-2 \sin 2 \psi \cos \omega_{\mathrm{a}} \mathrm{t}+2 \cos 2 \psi \cos \Delta \sin \omega_{\mathrm{a}} \mathrm{t} \\
& \mathrm{f}_{\triangle}=-\sin 2 \psi \sin \Delta \sin \omega_{\mathrm{a}} \mathrm{t} .
\end{aligned}
$$

From Eqs. (16) we see that each of the functions $f, f_{\psi}$, and $f_{\Delta}$ has only one harmonic and that their Fourier amplitudes evaluated at $\bar{\psi}, \bar{\Delta}$ are given by

$$
\begin{aligned}
& \mathrm{f}_{\mathrm{o}}=1, \mathrm{f}_{1} \sin \theta_{1}=\cos 2 \bar{\psi}, \mathrm{f}_{1} \cos \theta_{1}=\sin 2 \bar{\psi} \cos \bar{\Delta} ; \\
& \mathrm{f}_{\psi_{\mathrm{o}}}=0, \mathrm{f}_{\psi_{1}} \sin \theta_{\psi_{1}}=-2 \sin 2 \bar{\psi}, \mathrm{f}_{\psi_{1}} \cos \theta_{\psi_{1}}= \\
& 2 \cos 2 \bar{\psi} \cos \bar{\Delta} ;
\end{aligned}
$$

\footnotetext{
*Equation (12) follows directly from Eqs. (6) - (9) of Ref. 13 if we notice that $\left(1-|\chi|^{2}\right) /\left(1+|\chi|^{2}\right)=\cos 2 \psi, 2 \operatorname{Re}(\chi) /\left(1+|\chi|^{2}\right)=\sin 2 \psi \cos \Delta$, and $2 \operatorname{Im}(x) /\left(1+|\chi|^{2}\right)=\sin 2 \psi \sin \Delta$.
} 


$$
\mathrm{f}_{\Delta \mathrm{o}}=0, \mathrm{f}_{\triangle \mathrm{l}} \sin \theta_{\Delta \mathrm{l}}=0, \mathrm{f}_{\Delta \mathrm{l}} \cos \theta_{\Delta \mathrm{l}}=-\sin 2 \bar{\psi} \sin \bar{\Delta}
$$

To determine the average polarization $(\bar{\psi}, \bar{\Delta})$, we substitute from Eq. (17a) into Eq. (10a), where now $p=1$; this gives

$$
\eta^{\mathrm{c}} \omega_{\mathrm{a}}=\cos 2 \bar{\psi}, \eta^{\mathrm{s}} \omega_{\mathrm{a}}=\sin 2 \bar{\psi} \cos \bar{\Delta} \text {. }
$$

The first of Eq. (18) readily gives $\bar{\psi}$; from the second we subsequently obtain $\Delta$. The equations that determine the intensity and polarization modulation parameters $\delta \hat{\mathrm{I}} / \overline{\mathrm{I}}, \delta^{\hat{\psi}} \psi$ and $\hat{\delta} \Delta$ are obtained by substituting from Eqs. (17) into Eq. (11a), where p = 1 in the present case; they are

$$
\begin{aligned}
& \eta_{\mathrm{O}}^{\mathrm{e}}=(\hat{\delta \mathrm{I}} / \overline{\mathrm{I}}), \\
& \eta_{\omega_{\mathrm{a}}}^{\mathrm{ec}}=\cos 2 \bar{\psi}(\hat{\delta} \hat{\mathrm{I}} / \overline{\mathrm{I}})+(-2 \sin 2 \bar{\psi}) \hat{\psi} \psi, \\
& \eta_{\omega_{\mathrm{a}}}^{\mathrm{es}}=\sin 2 \bar{\psi} \cos \bar{\Delta}(\hat{\delta} \mathrm{I} / \overline{\mathrm{I}})+(2 \cos 2 \bar{\psi} \cos \bar{\Delta}) \hat{\delta} \hat{\psi}+ \\
& (-\sin 2 \bar{\psi} \sin \bar{\Delta}) \hat{\delta} \Delta .
\end{aligned}
$$

Equation (19a) gives $\hat{\delta I} / \bar{I}$; subsequently, $\delta \hat{\psi}$ and $\hat{\delta \Delta}$ are readily determined from Eqs. (19b) and (19c), respectively.

\section{Rotating Half-Wave Plate and \\ Fixed Linear Analyzer (RHWP+FLA)}

When the element $\mathrm{E}$ acts as a half-wave plate (HWP), we set $\delta=$ $\pi$ in Eq. (14) to obtain

$$
f=1+\cos 2 \psi \cos (2 A-4 \theta)-\sin 2 \psi \cos \Delta \sin (2 A-4 \theta) \text {. }
$$

Furthermore, if we assume that the linear analyzer (LA) is positioned at zero azimuth, $\mathrm{A}=0$, and that the HWP is rotating at constant angular speed of $1 / 4 \omega_{a}$, so that $\theta=\omega_{a} t / 4$, Eq. (20) becomes

$$
\mathrm{f}=1+\cos 2 \psi \cos \omega_{\mathrm{a}} \mathrm{t}+\sin 2 \psi \cos \Delta \sin \omega_{\mathrm{a}} \mathrm{t} .
$$

Equation (21) is identical with Eq. (16a) for the case of a rotating linear analyzer alone. Thus, the analysis of Section III, including all of Eqs. (16) - (19), applies to the RHWP+FLA.

The advantage of using the RHWP+FLA over the RLA is that the state of polarization of light that leaves the analyzer is constant in the former case and variable in the latter. Therefore, systematic errors due to a polarization-dependent photo-electric response are avoided. The disadvantage, of course, is the addition of another optical component.

\section{Rotating Quarter-Wave Plate and Fixed Linear Analyzer (RQWP+FLA)}

When the element $\mathrm{E}$ acts as a quarter-wave plate (QWP), we set $\delta=\pi / 2$ in Eq. (14) to obtain

$$
\begin{aligned}
\mathrm{f}=1 & +\cos 2 \psi \cos 2 \theta \cos (2 \mathrm{~A}-2 \theta)+\sin 2 \psi \cos \Delta \sin 2 \theta \cos (2 \mathrm{~A}-2 \theta) \\
& +\sin 2 \psi \sin \Delta \sin (2 \mathrm{~A}-2 \theta) .
\end{aligned}
$$

If we substitute $A=0$ and $\theta=\omega_{a} t / 2$ in Eq. (22), and take the first partial derivatives of the result with respect to $\psi$ and $\Delta$, we obtain

$$
\begin{aligned}
& \mathrm{f}=(1+1 / 2 \cos 2 \psi)+(-\sin 2 \psi \sin \Delta) \sin \omega_{\mathrm{a}} \mathrm{t}+ \\
& (1 / 2 \sin 2 \psi \cos \Delta) \sin 2 \omega_{\mathrm{a}} \mathrm{t}+(1 / 2 \cos 2 \psi) \cos 2 \omega_{\mathrm{a}} \mathrm{t} \\
& \mathrm{f}_{\psi}=(-\sin 2 \psi)+(-2 \cos 2 \psi \sin \Delta) \sin \omega_{\mathrm{a}} \mathrm{t}+ \\
& (\cos 2 \psi \cos \Delta) \sin 2 \omega_{\mathrm{a}} \mathrm{t}+(-\sin 2 \psi) \cos 2 \omega_{\mathrm{a}} \mathrm{t} \\
& \mathrm{f}_{\triangle}=(-\sin 2 \psi \cos \Delta) \sin \omega_{\mathrm{a}} \mathrm{t}+(-1 / 2 \sin 2 \psi \sin \Delta) \sin 2 \omega_{\mathrm{a}} \mathrm{t}
\end{aligned}
$$

Equations (23) show that each of the functions $f, f_{\psi}$ and $f_{\triangle}$ for the RQWP+FLA system possesses two spectral components (of frequencies $\omega_{\mathrm{a}}$ and $2 \omega_{\mathrm{a}}$ ), in addition to the dc component. The average polarization can be determined from the normalized amplitudes of the $\cos 2 \omega_{\mathrm{a}} \mathrm{t}$ and $\sin 2 \omega_{\mathrm{a}} \mathrm{t}$ components of the baseband signal $\mathrm{S}_{\mathrm{bb}}$ by substituting from Eq. (23a) into Eq. (10a), where $\mathrm{p}=2$; this gives

$$
\begin{aligned}
& \eta_{2}^{\mathrm{c}}{ }_{2} \omega_{\mathrm{a}}=1 / 2 \cos 2 \bar{\psi} /(1+1 / 2 \cos 2 \bar{\psi}), \eta_{2}^{\mathrm{s}} \omega_{\mathrm{a}}= \\
& 1 / 2 \sin 2 \bar{\psi} \cos \bar{\Delta} /(1+1 / 2 \cos 2 \bar{\psi}) .
\end{aligned}
$$

The first of Eq. (24) can be readily solved for $\cos 2 \bar{\psi}$, hence for $\bar{\psi}$, and $\bar{\Delta}$ is subsequently determined from the second. The modulation parameters can be obtained from the normalized dc and the normalized amplitudes of the $\cos 2 \omega_{\mathrm{a}} t$ and $\sin 2 \omega_{\mathrm{a}} \mathrm{t}$ components of the envelope signal $\delta \mathrm{S}_{\mathrm{e}}$ by substituting from Eqs. (23b) and $(23 \mathrm{c})$ into Eq. (11a), where $\mathrm{p}=2$; this gives

$$
\begin{aligned}
& \mathrm{f}_{\mathrm{o}} \eta_{\mathrm{O}}^{\mathrm{e}}=\mathrm{f}_{\mathrm{o}}(\hat{\delta \mathrm{I}} / \overline{\mathrm{I}})+(-\sin 2 \bar{\psi}) \hat{\delta} \psi \\
& \mathrm{f}_{\mathrm{o}} \eta_{2}^{\mathrm{ec}} \omega_{\mathrm{a}}=(1 / 2 \cos 2 \bar{\psi})(\hat{\delta \mathrm{I} / \overline{\mathrm{I}})})+(-\sin 2 \bar{\psi}) \hat{\delta}, \\
& \mathrm{f}_{\mathrm{o}} \eta_{2}^{\mathrm{es}} \omega_{\mathrm{a}}=(1 / 2 \sin 2 \bar{\psi} \cos \bar{\Delta})(\hat{\delta} \mathrm{I} / \overline{\mathrm{I}})+(\cos 2 \bar{\psi} \cos \bar{\Delta}) \hat{\delta} \psi+ \\
& (-1 / 2 \sin 2 \bar{\psi} \sin \bar{\Delta}) \hat{\delta} \Delta \text {, } \\
& \mathrm{f}_{\mathrm{O}}=1+1 / 2 \cos 2 \bar{\psi} \text {. }
\end{aligned}
$$

Equations (25a) and (25b) can be readily solved for $\hat{\delta} \mathrm{I} / \overline{\mathrm{I}}$ and $\hat{\delta} \hat{\psi}$, and subsequently $\delta \Delta$ is determined by Eq. $(25 \mathrm{c})$.

\section{Rotating Linear Analyzer and Fixed Linear Analyzer (RLA+FLA)}

If we replace the retardation plate $E$ in Figure 1 by a second linear analyzer $\mathrm{LA}^{\prime}$ of azimuth $\mathrm{A}^{\prime}$, the transmission function $\mathrm{f}$ for the two analyzers can be written as $f=f_{A^{\prime}} \cdot f_{A^{\prime}}$, $A$ where $f_{A^{\prime}}$ describes the effect of $\mathrm{LA}^{\prime}$ only and $\mathrm{f}_{\mathrm{A}^{\prime}}$, $\mathrm{A}$ describes the transmission of LA for the linearly polarized light leaving $L^{\prime}{ }^{\prime} . f_{A^{\prime}}$ is given by $\mathrm{Eq}$. (15) with $\mathrm{A}=\mathrm{A}^{\prime}$ and $\mathrm{f}_{\mathrm{A}^{\prime}, \mathrm{A}}$ is given by $\cos ^{2}\left(\mathrm{~A}^{\prime}-\mathrm{A}\right)$, according to the well-known law of Malus. Therefore, we can readily write

$$
f=\left[1+\cos 2 \psi \cos 2 A^{\prime}+\sin 2 \psi \cos \Delta \sin 2 A^{\prime}\right]\left[1+\cos \left(2 A^{\prime}-2 A\right)\right] .
$$

When LA is fixed at zero azimuth and $\mathrm{LA}^{\prime}$ is rotating at constant angular speed $\omega_{a} / 2$, we substitute $A=0, A^{\prime}=1 / 2 \omega_{a} t$ in Eq. (26) to get

$$
\begin{aligned}
& \mathrm{f}=(1+1 / 2 \cos 2 \psi)+(\sin 2 \psi \cos \Delta) \sin \omega_{\mathrm{a}} \mathrm{t}+(1+\cos 2 \psi) \cos \omega_{\mathrm{a}} \mathrm{t}+ \\
& (1 / 2 \sin 2 \psi \cos \Delta) \sin 2 \omega_{\mathrm{a}} \mathrm{t}+(1 / 2 \cos 2 \psi) \cos 2 \omega_{\mathrm{a}} \mathrm{t} \\
& \mathrm{f}_{\psi}=(-\sin 2 \psi)+(2 \cos 2 \psi \cos \Delta) \sin \omega_{\mathrm{a}} \mathrm{t}+(-2 \sin 2 \psi) \cos \omega_{\mathrm{a}} \mathrm{t}+ \\
& (\cos 2 \psi \cos \Delta) \sin 2 \omega_{\mathrm{a}} \mathrm{t}+(-\sin 2 \psi) \cos 2 \omega_{\mathrm{a}} \mathrm{t} \\
& \mathrm{f}_{\triangle}=(-\sin 2 \psi \sin \Delta) \sin \omega_{\mathrm{a}} \mathrm{t}+(-1 / 2 \sin 2 \psi \sin \Delta) \sin 2 \omega_{\mathrm{a}} \mathrm{t}
\end{aligned}
$$

where $f_{\psi}$ and $f_{\triangle}$ are obviously obtained from $f$ by partial differentiation. The average polarization can be obtained from the amplitudes of the cosine and sine terms of either the $\omega_{\mathrm{a}}$ or the $2 \omega_{\mathrm{a}}$ component of $S_{\mathrm{bb}}$ by substituting from Eq. (27a) into Eq. (10a). If the $\omega_{\mathrm{a}}$ component is selected, we obtain

$$
\begin{aligned}
& \eta_{\omega_{\mathrm{a}}}^{\mathrm{c}}=(1+\cos 2 \bar{\psi}) /(1+1 / 2 \cos 2 \bar{\psi}), \eta_{\omega_{\mathrm{a}}}^{\mathrm{s}}= \\
& \sin 2 \bar{\psi} \cos \bar{\Delta} /(1+1 / 2 \cos 2 \bar{\psi}),
\end{aligned}
$$

and if the $2 \omega_{\mathrm{a}}$ component is used instead, we get

$$
\eta_{2}^{\mathrm{c}} \omega_{\mathrm{a}}=1 / 2 \cos 2 \bar{\psi} /(1+1 / 2 \cos 2 \bar{\psi}), \eta_{2}^{\mathrm{s}} \omega_{\mathrm{a}}=
$$


$1 / 2 \sin 2 \bar{\psi} \cos \bar{\Delta} /(1+1 / 2 \cos 2 \bar{\psi})$

The first of either Eqs. (28a) or (28b) can be readily solved for $\cos 2 \bar{\psi}$, hence for $\bar{\psi}$, and from the second we next get $\bar{\Delta}$. The modulation parameters $\delta \hat{\mathrm{I}} / \overline{\mathrm{I}}, \delta \hat{\psi}$ and $\delta \Delta$ can be obtained from the dc component and the amplitudes of the cosine and sine terms of either the $\omega_{\mathrm{a}}$ or the $2 \omega_{\mathrm{a}}$ components of the envelope signal $\delta \mathrm{S}_{\mathrm{e}}$ by substituting from Eqs. (27b) and (27c) into Eq. (11a); this gives

$$
\begin{aligned}
& \mathrm{f}_{\mathrm{o}} \eta^{\mathrm{e}}{ }_{\mathrm{o}}=\mathrm{f}_{\mathrm{o}}(\hat{\delta \mathrm{I}} / \overline{\mathrm{I}})+(-\sin 2 \bar{\psi}) \hat{\delta} \psi \\
& \mathrm{f}_{\mathrm{o}} \eta_{\omega_{\mathrm{a}}}^{\mathrm{ec}}=(1+\cos 2 \bar{\psi})(\hat{\delta \mathrm{I} / \overline{\mathrm{I}}})+(-2 \sin 2 \bar{\psi}) \hat{\delta} \text {, } \\
& \mathrm{f}_{\mathrm{o}} \eta_{\omega_{\mathrm{a}}}^{\mathrm{es}}=(\sin 2 \bar{\psi} \cos \bar{\Delta})(\hat{\delta} \mathrm{I} / \overline{\mathrm{I}})+(2 \cos 2 \bar{\psi} \cos \bar{\Delta}) \hat{\delta} \hat{\psi}+ \\
& (-\sin 2 \bar{\psi} \sin \bar{\Delta}) \hat{\delta} \Delta \text {, } \\
& \mathrm{f}_{\mathrm{o}} \eta_{2}^{\mathrm{ec}} \omega_{\mathrm{a}}=(1 / 2 \cos 2 \bar{\psi})(\hat{\delta} \mathrm{I} / \overline{\mathrm{I}})+(-\sin 2 \bar{\psi}) \hat{\delta} \psi \\
& \mathrm{f}_{\mathrm{o}} \eta_{2}^{\mathrm{es}} \omega_{\mathrm{a}}=(1 / 2 \sin 2 \bar{\psi} \cos \bar{\Delta})(\hat{\delta} \mathrm{I} / \overline{\mathrm{I}})+(\cos 2 \bar{\psi} \cos \bar{\Delta}) \hat{\psi} \psi \\
& (-1 / 2 \sin 2 \bar{\psi} \sin \bar{\Delta}) \hat{\delta} \Delta \text {, } \\
& \mathrm{f}_{\mathrm{O}}=1+1 / 2 \cos 2 \bar{\psi} \text {. }
\end{aligned}
$$

From Eq. (29a) and either (29b) or (29d), we solve for $\hat{\delta} \mathbf{I} / \bar{I}$ and $\delta \hat{\psi}$. Next, we obtain $\delta \Delta$ from Eq. (29c) or (29e), respectively.

It should be noted that the advantage of adding one more analyzer in the RLA+FLA system, as compared to the RLA system, is that the photodetector sees light of constant polarization, hence errors due to possible polarization-dependent photoelectric response are avoided. Furthermore, the two available frequency components provide independent determinations of the polarization parameters and can be expolited for improving the accuracy of measurements, or for providing internal-consistency checks.

\section{Oscillating-Phase Retarder and Fixed Linear Analyzer (OPhR+FLA)}

If we go back to the two-element periodic analyzer E+LA of Section II and substitute $\theta=0$ in to Eq. (14), we get

$$
\begin{aligned}
& \mathrm{f}=1+\cos 2 \psi \cos 2 \mathrm{~A}+\sin 2 \psi \cos \Delta \sin 2 \mathrm{~A} \cos \delta+ \\
& \sin 2 \psi \sin \Delta \sin 2 \mathrm{~A} \sin \delta,
\end{aligned}
$$

which corresponds to a retarder of arbitrary phase $\delta$, set at zero azimuth, which is followed by a linear analyzer of azimuth A. If we further set $A=\pi / 4$ and $\delta=\hat{\delta} \sin \omega_{\mathrm{a}} \mathrm{t}$ (as is obtained by an oscillating piezo- or electro-optic birefringence), Eq. (30) becomes

$$
f=1+\sin 2 \psi \cos \Delta \cos \left(\hat{\delta} \sin \omega_{a} t\right)+\sin 2 \psi \sin \Delta \sin \left(\hat{\delta} \sin \omega_{a} t\right) .
$$

We next expand $\cos \left(\hat{\delta} \sin \omega_{15} \mathrm{t}\right)$ and $\sin \left(\hat{\delta} \sin \omega_{\mathrm{a}} \mathrm{t}\right)$ in Eq. (31) using the Bessel-function series ${ }^{15}$

$$
\begin{aligned}
& \cos (X \sin \omega t)=\left[J_{0}(X)+2 \sum_{n=1}^{\infty} J_{2 n}(X) \cos (2 n \omega t)\right], \\
& \sin (X \sin \omega t)=2 \sum_{n=0}^{\infty} J_{2 n+1}(X) \sin [(2 n+1) \omega t]
\end{aligned}
$$

and retain only the fundamental and second-harmonic components; this gives

$$
\begin{aligned}
& \mathrm{f}=\left[1+\sin 2 \psi \cos \Delta \mathrm{J}_{\mathrm{o}}(\hat{\delta})\right]+\left[2 \sin 2 \psi \sin \Delta \mathrm{J}_{1}(\hat{\delta})\right] \sin \omega_{\mathrm{a}} \mathrm{t}+ \\
& {\left[2 \sin 2 \psi \cos \Delta \mathrm{J}_{2}(\hat{\delta})\right] \cos 2 \omega_{\mathrm{a}} \mathrm{t}+\ldots}
\end{aligned}
$$

$$
\begin{aligned}
& \mathrm{f}_{\psi}=\left[2 \cos 2 \psi \cos \Delta \mathrm{J}_{\mathrm{o}}(\hat{\delta})\right]+\left[4 \cos 2 \psi \sin \Delta \mathrm{J}_{1}(\hat{\delta})\right] \sin \omega_{\mathrm{a}} \mathrm{t}+ \\
& {\left[4 \cos 2 \psi \cos \Delta \mathrm{J}_{2}(\hat{\delta})\right] \cos 2 \omega_{\mathrm{a}} \mathrm{t}+\ldots} \\
& \mathrm{f}_{\triangle}=\left[-\sin 2 \psi \sin \Delta \mathrm{J}_{\mathrm{o}}(\hat{\delta})\right]+\left[2 \sin 2 \psi \cos \mathrm{J}_{1}(\hat{\delta})\right] \sin \omega_{\mathrm{a}} \mathrm{t}+ \\
& {\left[-2 \sin 2 \psi \sin \Delta \mathrm{J}_{2}(\hat{\delta})\right] \cos 2 \omega_{\mathrm{a}} \mathrm{t}+\ldots}
\end{aligned}
$$

The average polarization $(\bar{\psi}, \bar{\Delta})$ is determined from the normalized amplitudes of the $\omega_{\mathrm{a}}$ and $2 \omega_{\mathrm{a}}$ components of $\mathrm{S}_{\mathrm{bb}}$ by substituting from Eq. (33a) into Eq. (10b); this yields

$$
\begin{aligned}
& \eta_{\omega_{\mathrm{a}}}=2 \sin 2 \bar{\psi} \sin \bar{\Delta} \mathrm{J}_{1}(\hat{\delta}) /\left[1+\sin 2 \bar{\psi} \cos \bar{\Delta} \mathrm{J}_{\mathrm{O}}(\hat{\delta})\right], \\
& \eta_{2 \omega_{\mathrm{a}}}=2 \sin 2 \bar{\psi} \cos \bar{\Delta} \mathrm{J}_{2}(\hat{\delta}) /\left[1+\sin 2 \bar{\psi} \cos \bar{\Delta} \mathrm{J}_{\mathrm{O}}(\hat{\delta})\right]
\end{aligned}
$$

$\bar{\Delta}$ can be easily obtained by taking the ratio of the two of Eqs. (34) yielding $\tan \bar{\Delta}$; then $\psi$ is derived from either one of these equations.

The modulation parameters are determined from the normalized amplitudes of the carrier $\left(\omega_{m}\right)$ and the first two sidebands $\left(\omega_{\mathrm{m}} \pm \omega_{\mathrm{a}}, \omega_{\mathrm{m}} \pm 2 \omega_{\mathrm{a}}\right)$ of $\delta \mathrm{S}_{\mathrm{mc}}$ by substituting from Eqs. (33) into Eq. $(11 \mathrm{a})$; this gives

$$
\begin{aligned}
& \mathrm{f}_{\mathrm{o}} \eta_{\omega_{\mathrm{m}}}=\mathrm{f}_{\mathrm{o}}(\hat{\delta \mathrm{I} /} / \overline{\mathrm{I}})+\left[2 \cos 2 \bar{\psi} \cos \bar{\Delta} \mathrm{J}_{\mathrm{o}}(\hat{\delta})\right] \delta \hat{\psi}+ \\
& {\left[-\sin 2 \bar{\psi} \sin \bar{\Delta} \mathrm{J}_{\mathrm{o}}(\hat{\delta})\right] \delta \Delta,} \\
& 2 \mathrm{f}_{\mathrm{o}} \eta_{\omega_{\mathrm{m}}} \pm \omega_{\mathrm{a}}=\left[2 \sin 2 \bar{\psi} \sin \bar{\Delta} \mathrm{J}_{1}(\hat{\delta})\right](\hat{\delta} \mathrm{I} / \overline{\mathrm{I}})+ \\
& {\left[4 \cos 2 \bar{\psi} \sin \bar{\Delta} \mathrm{J}_{1}(\hat{\delta})\right] \delta \hat{\psi}+\left[2 \sin 2 \bar{\psi} \cos \bar{\Delta} \mathrm{J}_{1}(\hat{\delta})\right] \delta \Delta} \\
& 2 \mathrm{f}_{\mathrm{o}} \eta_{\omega_{\mathrm{m}}} \pm 2 \omega_{\mathrm{a}}=\left[2 \sin 2 \bar{\psi} \cos \bar{\Delta} \mathrm{J}_{2}(\hat{\delta})\right](\hat{\delta} \mathrm{I} / \overline{\mathrm{I}})+ \\
& {\left[4 \cos 2 \bar{\psi} \cos \bar{\Delta} \mathrm{J}_{2}(\hat{\delta})\right] \delta \hat{\psi}+\left[-2 \sin 2 \bar{\psi} \sin \bar{\Delta} \mathrm{J}_{2}(\hat{\delta})\right] \delta \Delta,} \\
& \mathrm{f}_{\mathrm{o}}=1+\sin 2 \bar{\psi} \cos \bar{\Delta} \mathrm{J}_{\mathrm{O}}(\hat{\delta}),
\end{aligned}
$$

which can be solved simultaneously for $\hat{\delta I} / \bar{I}, \hat{\delta} \psi$ and $\hat{\delta \Delta}$.

\section{Oscillating Optical Rotator and Fixed Linear Analyzer (OOR+FLA)}

The effect of adding an optical rotator (OR), that gives a rotation $\mathrm{r}$, before a linear analyzer of azimuth $\mathrm{A}$ is to change the function $f$ of Eq. (15) to

$$
f=1+\cos 2 \psi \cos (2 A+2 r)+\sin 2 \psi \cos \Delta \sin (2 A+2 r)
$$

If we choose $A=0$ and $r=\hat{r} \sin \omega_{a} t$ (obtained, e.g., by an acexcited Faraday cell), Eq. (36) becomes

$$
f=1+\cos 2 \psi \cos \left(\hat{r} \sin \omega_{a} t\right)+\sin 2 \psi \cos \Delta \sin \left(\hat{r} \sin \omega_{a} t\right)
$$

Following a procedure exactly similar to that used in Section VII, we find that the average polarization is given by

$$
\begin{aligned}
& \eta_{\omega_{\mathrm{a}}}=2 \sin 2 \bar{\psi} \cos \bar{\Delta} \mathrm{J}_{1}(\hat{\mathrm{r}}) /\left[1+\cos 2 \bar{\psi} \mathrm{J}_{\mathrm{o}}(\hat{\mathrm{r}})\right], \\
& \eta_{2 \omega_{\mathrm{a}}}=2 \cos 2 \bar{\psi} \mathrm{J}_{2}(\hat{\mathrm{r}}) /\left[1+\cos 2 \bar{\psi}_{\mathrm{o}}(\hat{\mathrm{r}})\right] ;
\end{aligned}
$$

while the modulation parameters are given by

$$
\begin{aligned}
& \mathrm{f}_{\mathrm{o}} \eta_{\omega_{\mathrm{m}}}=\mathrm{f}_{\mathrm{o}}(\hat{\mathrm{I}} / \overline{\mathrm{I}})+\left[-2 \sin 2 \bar{\psi} \mathrm{J}_{\mathrm{o}}(\hat{\mathrm{r}})\right] \delta \hat{\psi}, \\
& 2 \mathrm{f}_{\mathrm{o}} \eta_{\omega_{\mathrm{m}}} \pm \omega_{\mathrm{a}}=\left[2 \sin 2 \bar{\psi} \cos \overline{\mathrm{J}}_{1}(\hat{\mathrm{r}})\right](\hat{\delta} \mathrm{I} / \overline{\mathrm{I}})+ \\
& {\left[4 \cos 2 \bar{\psi} \cos \bar{\Delta} \mathrm{J}_{1}(\hat{\mathrm{r}})\right] \delta \hat{\psi}+\left[-2 \sin 2 \bar{\psi} \sin \bar{\Delta} \mathrm{J}_{1}(\hat{\mathrm{r}})\right] \delta \Delta,} \\
& 2 \mathrm{f}_{\mathrm{o}} \eta_{\omega_{\mathrm{m}}} \pm 2 \omega_{\mathrm{a}}=\left[2 \cos 2 \bar{\psi} \mathrm{J}_{2}(\hat{\mathrm{r}})\right](\hat{\delta} \mathrm{I} / \overline{\mathrm{I}})+ \\
& {\left[-4 \sin 2 \bar{\psi} \mathrm{J}_{2}(\hat{\mathrm{r}})\right] \delta \hat{\psi},} \\
& \mathrm{f}_{\mathrm{O}}=1+\cos 2 \bar{\psi}_{\mathrm{o}}(\hat{\mathrm{r}}) .
\end{aligned}
$$


Notice that the second of Eqs. (38) gives $\cos 2 \bar{\psi}$, hence $\bar{\psi}$, and that $\bar{\Delta}$ can be next determined from the first equation. Equations (39a) and (39c) can also be easily solved for $\delta \hat{I} / \bar{I}$ and $\delta \hat{\psi}$ and subsequently $\delta \triangle$ is obtained from Eq. (39b).

\section{Conclusion}

We have obtained relations that allow the use of six different periodic analyzers for the frequency-mixing detection of polarization- (and intensity-) modulated light. In all cases, the average polarization as well as the intensity- and polarization-modulation parameters of the beam are explicitly determined from limited Fourier analysis of the detected signal.

\section{References}

1. See, for example, W. K. Pratt, Laser Communication Systems, Wiley, 1969.

2. See, for example, M. Cardona, Modulation Spectroscopy, Academic, 1969.

3. R. M. A. Azzam "Frequency-Mixing detection (FMD) of polarization modulated light," J. Opt. Soc. Am., Vol. 66, 735-739 (1976).

4. R. M. A. Azzam and N. M. Bashara, "Polarization transfer function of an optical system as a bilinear transformation," J. Opt. Soc. Am., Vol. 62, 222-229 (1972).
5. B. D. Cahan and R. F. Spanier, "A high speed precision ellipsometer," Surface Sci., Vol. 16, 166-176 (1969).

6. D. E. Aspnes, "Fourier transform detection system for rotating analyzer ellipsometer," Opt. Commun., Vol. 8, 222-225 (1973).

7. P. S. Hauge and F. H. Dill, "Design and operation of ETA, an automated ellipsometer," IBM J. Res. Devel., Vol. 17, $472-489$ (1973).

8. Y. J. van der Meulen and N. C. Hien, "Design and operation of an automated high-temperature ellipsometer," J. Opt. Soc. Am., Vol. $64,804-811,(1974)$.

9. D. Clarke and R. N. Ibbett, "Three channel astronomical photoelectric spectropolarimeter," J. Phys. E: Sci. Instrum. Vol. 1, 409-411 (1968).

10. P. S. Hauge and F. H. Dill, "A rotating compenstator Fourier ellipsometer," Opt. Commun., Vol. 14, 431-435 (1975).

11. R. W. Stobie, B. Rao, and M. J. Dignam "Analysis of a novel ellipsometer technique for infrared spectroscopy," J. Opt. Soc. Am., Vol. 65, 25-28 (1975)

12. B. A. Ioshpa and V. N. Obridko, "Photoelectric analysis of polarized light," Opt. Spectrosc., Vol. 15, 60-62 (1963).

13. R. M. A. Azzam, "Alternate arrangement and analysis of systematic errors for dynamic photometric ellipsometers employing an oscillating-phase retarder," Optik, Vol. 45, 209-218 (1976).

14. R. M. A. Azzam, "Oscillating-analyzer ellipsometer," Rev. Sci. Instrum., Vol. 47, 624-628 (1976).

15. M. Abramowitz and I. A. Stegun editors, Handbook of Mathematical functions, Dover, 1965. 